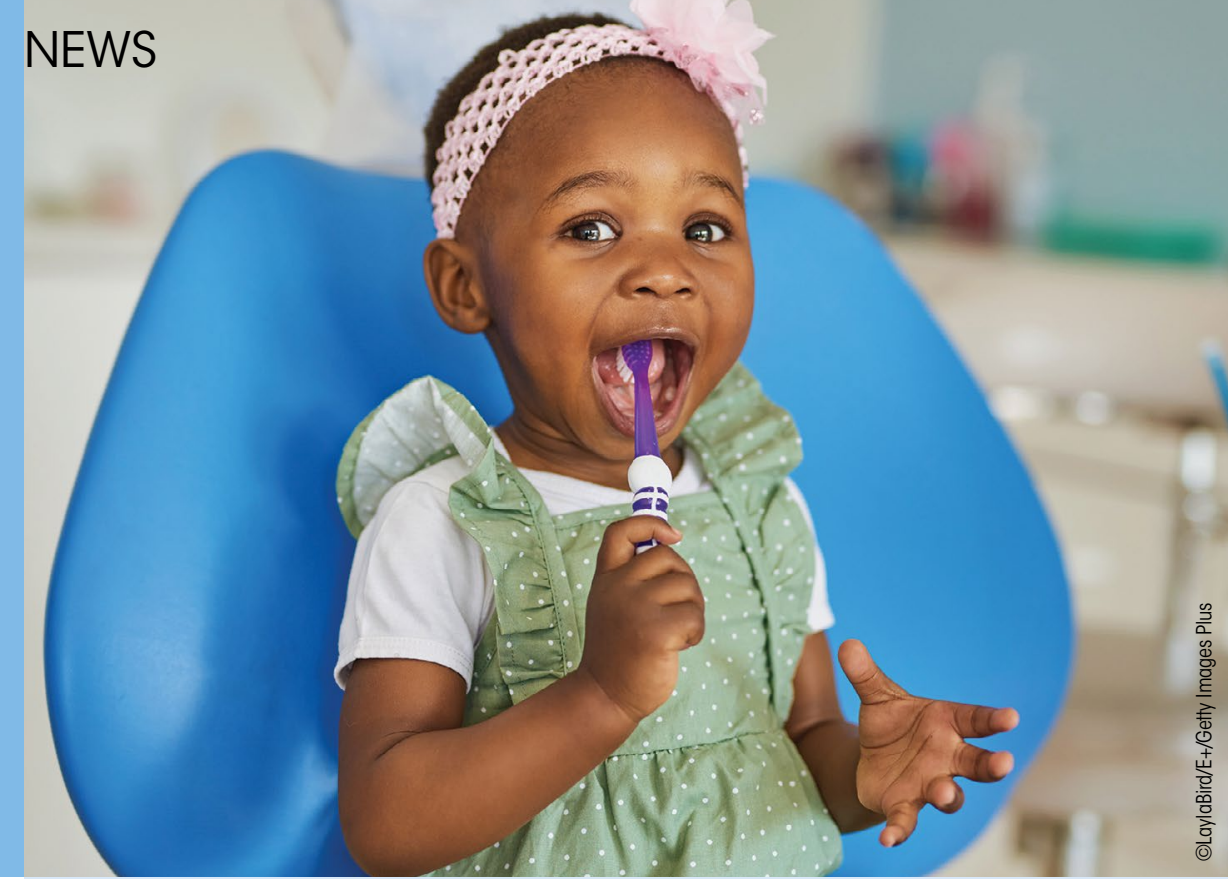

\title{
ORAL HEALTH OMITTED FROM EARLY YEARS REVIEW
}

he omission of oral health from 'The
Best Start for Life', The Early Years Healthy Development Review led by Andrea Leadsom, ${ }^{1}$ has been condemned by the British Society of Paediatric Dentistry (BSPD).

The newly published report provides a vision for the first critical 1,001 days of a child's life. Commissioned by Boris Johnson to improve the health and development outcomes for babies in England, there is scant mention of the mouth or of dentistry.

Claire Stevens CBE, BSPD spokesperson, said: 'This is a report which appears to deny the importance of oral health to the health and wellbeing of the nation's children. It's hugely frustrating that the contribution of paediatric dentists has not been sought despite an offer to pull together an expert group, making this a missed opportunity.'

The word 'dentist' is used once in the report.

Dr Stevens said: 'With childhood dental extractions under general anaesthetic being the most common reason for a child to be admitted to hospital, this omission is breath-taking. The report is 147 pages, yet there is sadly not a single mention of children's oral health.

'I am urging Andrea Leadsom and the Government to take up our offer and engage with paediatric dentists so that in the coming months, the oral health needs of children can be factored into the vision for a child's first 1,001 days.'

BSPD's data show that COVID-19 has had a disproportionate effect on young children and those from a lower socioeconomic groups and waiting lists for general anaesthetics are unacceptably long.

BSPD has been urging Government to ensure that oral health is included in the review. A Dental Check by the age of One, for instance, gets children into the routine of seeing a dentist and gives parents access to all-important oral health and dietary advice.

While the pandemic was a barrier to routine dental visits, the BSPD believes that parents need to be encouraged to engage with dentistry and caring for their children's teeth.

The mouth is factored into the report under the heading of infant feeding with a brief reference to tongue-tie.

Dr Stevens said: 'We welcome the inclusion of tongue-tie. That any baby should be struggling to breastfeed due to tongue-tie is worrying. What we would like to see is a mouth check included as routine in the newborn check on every baby before they leave hospital so tonguetie can be picked up early'.

\section{Reference}

1. HM Government. The Best Start for Life. A Vision for the 1,001 Critical Days. The Early Years Healthy Development Review Report. 2021. Available at: https://assets.publishing. service.gov.uk/government/uploads/ system/uploads/attachment_data/ file/973112/The_best_start_for_life_a_ vision_for_the_1_001_critical_days.pdf (accessed 27 March 2021).
GDC introduces option to

\section{pay ARF by \\ instalments}

The General Dental Council (GDC) has announced a new pay by instalments option for the annual retention fee (ARF), designed to support dental professionals who pay their own fee by enabling them to spread the cost over the year with quarterly direct debits. For dental care professionals (DCPs) this works out as four payments of $£ 28.50$.

DCPs need to log in to eGDC from early May and select the quarterly direct debit option no later than 31 May 2021. The regulator is working to get this new option set up and says it will be in touch with DCPs when they are able to sign up.

GDC Chief Executive and Registrar, Ian Brack, said: 'We know that many dental professionals want more flexibility in how they pay the ARF. Work is underway to make the necessary changes to our systems to allow us to offer this to DCPs this year. We expect this work to be complete by early May and DCPs who want to pay quarterly will then need to sign up by 31 May. Detailed guidance will follow nearer the time.

The British Society of Dental Hygiene and Therapy (BSDHT) and the British Association of Dental Nurses (BADN) have welcomed the GDC's decision.

BSDHT commented that it was glad the GDC had listened to the concerns of BSDHT and its members, and made the more flexible option available. The society hopes this measure will make a difference for many dental hygienists and therapists in the future.

BADN President Jacqui Elsden said: 'BADN have been lobbying the GDC for some years to allow payment by instalments, but the answer was always that this would be too costly, and additionally that it was not possible under the current legal framework'.

BADN believe that $£ 114$ a year is still too high a fee for dental nurses.

Ms Elsden said: 'We hope that now the GDC have acknowledged the financial burden the ARF places on dental nurses, they will give further consideration to BADN's two other proposals: that the ARF be lowered to a more realistic figure for dental nurses, and that a lower ARF be implemented for all dental professionals, not just dental nurses, who work part time'. 